doi: 10.25005/2074-0581-2021-23-2-242-250

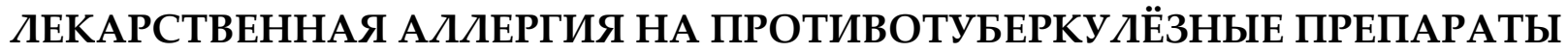 У БОЛЬНЫХ ТУБЕРКУ ЯЁЗОМ С МНОЖЕСТВЕННОЙ ЯЕКАРСТВЕННОЙ УСТОЙЧИВОСТЬЮ
}

\author{
Б.Б. МАЙКАНАЕВ, А.А. ТОКТОГОНОВА, Е.В. ДУДЕНКО, С. СЫДЫКОВА
}

Национальный центр фтизиатрии, Бишкек, Кыргызская Республика

\begin{abstract}
Цель: выявление аллергической реакции (АР) на противотуберкулёзные препараты (ПтП) у больных туберкулёзом с множественной лекарственной устойчивостью (МЛУ ТБ).

Материал и методы: выполнено выявление АР на ПТП у 50 больных МлУ ТБ лёгких с подозрением на наличие лекарственной аллергии. Пациенты получали лечение по стандартной схеме химиотерапии. Использовалась реакция сенсибилизации лизиса лейкоцитов (РСЛл) для выявления гиперчувствительности замедленного типа. Материалом для исследования служили пробы периферической крови. Всего по 9 ПТП - изониазиду (H), пиразинамиду (Z), этамбутолу (E), левофлоксацину (Lfx), циклосерину (Cs), протионамиду (Pt), каприомицину (Cm), ПАСК (PAS) и пиридоксину $\left(\mathrm{B}_{6}\right)$ - выполнено 331 исследование. Дизайн исследования: ретроспективный.

Результаты: положительные АР отмечены у 78,0\%, слабоположительные - у 20,0\%, отрицательные - у 2,0\% из 50 больных. Анализ частоты AP у больных МлУ ТБ показал, что на Cs (60,0\%), Pt (52,1\%), PAS (52,8\%), H (54,5\%), Lfx (52,2\%) развитие AP было чаще, чем на другие ПТП. AP на $\mathrm{Cm}(38,3 \%), \mathrm{Z}(37,8 \%)$, E (41,0\%) и В $(38,7 \%)$ встречались примерно с одинаковой частотой. Наиболее часто наблюдались АР к 2 ПтП у 12 $(24,0 \%)$ пациентов.

Заключение: АР выявлены у 98,0\% из 50 обследованных больных МлУ ТБ с подозрением на лекарственную аллергию. Наиболее аллергенным препаратом является циклосерин (60,0\% лизиса лейкоцитов). Количество больных МлУ ТБ с АР к 2 ПтП составило 24,0\% пациентов. Ключевые слова: МЛУТБ, лекарственная устойчивость, противотуберкулёзные химиопрепараты, аллергические реакции.

Для цитирования: Майканаев ББ, Токтогонова АA, Дуденко EB, Сыдыкова СС. Лекарственная аллергия на противотуберкулёзные препараты у больных туберкулёзом с множественной лекарственной устойчивостью. Вестник Авиценны. 2021;23(2):242-50. Available from: https://doi. org/10.25005/2074-0581-2021-23-2-242-250
\end{abstract}

\section{ANTITUBERCULOSIS DRUG ALLERGY IN PATIENTS WITH MULTIDRUG-RESISTANT TUBERCULOSIS}

\section{B.B. MAYKANAEV, A.A. TOKTOGONOVA, E.V. DUDENKO, S. SYDYKOVA}

National Center for Phthisiology, Bishkek, Kyrgyz Republic

Objective: To identify allergic reactions (AR) to anti-tuberculosis drugs (ATD) in patients with multidrug-resistant tuberculosis (MDR-TB)

Methods: Total of 50 patients with MDR-TB, suspected to develop AR, were enrolled in the study. The patients received standard chemotherapy. The leukocyte lysis sensitization test (LLST) was used to detect delayed-type hypersensitivity. Peripheral blood samples were used to perform 331 tests for AR to nine ATD: isoniazid $(\mathrm{H})$, pyrazinamide $(\mathrm{Z})$, ethambutol (E), levofloxacin (Lfx), cycloserine (Cs), prothionamide (Pt), capreomycin (Cm), p-aminosalicylic acid(PAS) and pyridoxine $\left(B_{6}\right)$. Study design: retrospective.

Results: Tests for AR were positive in 39 patients (78.0\%), weakly positive - in 10 patients (20.0\%), negative - in 1 patient (2.0\%). Analysis of AR prevalence in MDR-TB patients showed that Cs (60.0\%), Pt (52.1\%), PAS (52.8\%), H (54.5\%), and Lfx (52.2\%) caused AR more frequently than the other ATD. AR to $\mathrm{Cm}$ (38.3\%), Z (37.8\%), E (41.0\%), and $B_{6}(38.7 \%)$ showed comparable prevalence. Most frequently the patients demonstrated AR to the two ATD (12 patients, 24.0\%).

Conclusion: AR were detected in $98.0 \%$ of 50 examined MDR-TB patients with suspected drug allergy (DA). Cs proved to be the most allergenic drug among others (60.0\%). Most frequently the MDR-TB patients demonstrated AR to the two ATD (24.0\% of patients).

Keywords: Multidrug-resistant tuberculosis, drug resistance, anti-tuberculosis chemotherapy drugs, allergic reactions.

For citation: Maykanaev BB, Toktogonova AA, Dudenko EV, Sydykova SS. Lekarstvennaya allergiya na protivotuberkulyoznye preparaty u bol'nykh tuberkulyozom s mnozhestvennoy lekarstvennoy ustoychivost'yu [Antituberculosis drug allergy in patients with multidrug-resistant tuberculosis]. Vestnik Avitsenny [Avicenna Bulletin]. 2021;23(2):242-50. Available from: https://doi.org/10.25005/2074-0581-2021-23-2-242-250

\section{ВВеДЕНИЕ}

По данным Всемирной организации здравоохранения (ВОЗ) в 2019 году в мире заболело туберкулёзом (ТБ) 10 миллионов человек, из этого числа 206030 человек заболели ТБ с множественной лекарственной устойчивостью микобактерий (МЛУ ТБ) [1]. Эффективность лечения у этой категории пациентов с применением традиционных режимов и препаратов составляет всего 54-55\%, по

\section{INTRODUCTION}

According to the World Health Organization, 10 million people worldwide developed tuberculosis (TB) in 2019, of which 206,030 people were diagnosed with MDR-TB [1]. The effectiveness of treatment in this category of patients using traditional treatment regimen and standard drugs is only $54-55 \%$, according to the world cohort analysis $[2,3]$. The efficacy of MDR-TB 
данным мирового когортного анализа [2, 3]. Эффективность лечения МЛУ ТБ в мире остаётся низкой, так как значительная доля пациентов прерывает лечение вследствие длительной по времени (до двух лет) терапии и возникновением нежелательных явлений (НЯ) от лекарственных препаратов [4]. Возникновение НЯ может привести к временному прерыванию или прекращению химиотерапии МЛУ ТБ [5-7].

Частота развития НЯ у больных МЛУ ТБ составляет $62-65 \%$ [8]. Как осложнение химиотерапии МЛУ ТБ препаратами второго ряда, НЯ встречались в 57,6\% случаев [9]. По другим данным, при химиотерапии МЛУ ТБ препаратами второго ряда наблюдалось 96\%-97\% нежелательных лекарственных реакций [10, 11]. При этом лекарственно-устойчивые формы микобактерий туберкулёза (МБТ) в большинстве случаев развиваются на фоне сниженного иммунитета [12].

В число наиболее распространённых НЯ входит лекарственная аллергия (ЛА). Согласно Международному Консенсусу по ЛА (International Consensus of Drug Allergy) реакции на лекарства, которые по клиническим признакам похожи на аллергию, обозначаются термином «реакции лекарственной гиперчувствительности» (РЛГ). ЛА - это РЛГ, для которой выявлен определённый иммунологический механизм [13]. Туберкулёз является инфекционно-аллергическим заболеванием, в его развитии участвует преимущественно IV тип аллергических реакций (AP) - клеточно-опосредованные реакции или РЛГ замедленного типа [14].

По некоторым данным, в группе больных МЛУ ТБ со стандартным режимом химиотерапии АР проявлялись в 13,5\% случаев [15]. Из числа 324 больных МЛУ ТБ с НЯ на ПТП второго ряда АР отмечены у 63 (19,7\%) [16]. Согласно другим источникам, при химиотерапии МЛУ ТБ, ЛА колебалась в широких пределах и составляла от $25 \%$ до $47,1 \%$ случаев [17]. В настоящее время не существует совершенно достоверных методов диагностики ЛА [18]. Таким образом, ЛА и иммунные механизмы ЛА при ТБ являются актуальной проблемой современной науки $[19,20]$.

\section{ЦЕЛЬ ИССЛЕДОВАНИЯ}

Выявление аллергической реакции (АР) на противотуберкулёзные препараты (ПтП) у больных туберкулёзом с множественной лекарственной устойчивостью (МЛУ ТБ).

\section{МАТЕРИАЛ И МЕТОДЫ}

Дизайн исследования: ретроспективное исследование. Для выполнения цели применён метод реакции сенсибилизации лизиса лейкоцитов (РСЛЛ). В качестве материала использовалась периферическая кровь больных МЛУ ТБ. Проведено обследование 50 больных МЛУ ТБ с подозрением на ЛА по клиническим данным. В группу больных МЛУ ТБ с признаками ЛА входили 23/46,0\% женщины и 27/54,0\% мужчин. Медиана возраста обследованных больных составила 34,9 (с диапазоном 19-66 лет). Пациенты находились на лечении в Национальном центре фтизиатрии в течение 2016-2018 годов. Данные больные принимали ПТП по стандартным схемам лечения. Использовался лабораторный метод определения IV или клеточно-опосредованного типа ЛА. Данный тест является провокационным тестом in vitro. Тест безопасен для пациента, так как применение предполагаемого аллергена и разрушение клеток крови пациента проходит вне организма. Создаются условия для проведения реакции (2 часа инкубации при $+37^{\circ} \mathrm{C}$ ). Результат оценивают методом световой микроскопии по степени лизиса лейкоцитов. Таким образом, происходит оценка возможности сенсибилизации пациента конкретными препаратами. treatment in the world remains low, since a significant share of patients interrupt treatment due to long-term (up to two years) therapy and the incidence of adverse drug events (AE) [4]. Development of $A E$ can lead to a temporary interruption or discontinuation of MDR-TB chemotherapy [5-7].

The prevalence of $A E$ in MDR-TB patients is $62-65 \%$ [8]. As a complication of MDR-TB chemotherapy with second-line drugs, $A E$ occurred in $57.6 \%$ of cases [9]. According to other data, $96 \%$ $97 \%$ of AE were observed during chemotherapy of MDR-TB with second-line drugs $[10,11]$. In these cases, drug-resistant forms of mycobacterium tuberculosis mostly develop on the background of weakened immune system [12].

The most common AE include DA. According to the International Consensus of DA, drug reactions that are clinically similar to allergies are termed drug hypersensitivity reactions (DHR). DA is a DHR for which a specific immunological mechanism has been identified [13]. TB is an infectious multisystemic disease, with type IV or delayed hypersensitivity mediated by cellular response involved in its pathogenesis [14].

According to some data, in a group of MDR-TB patients receiving a standard chemotherapy regimen, $A R$ were manifested in $13.5 \%$ of cases [15]. Of the 324 MDR-TB patients with AE to the second-line ATD, AR were observed in 63 (19.7\%) [16]. According to other sources, during chemotherapy for MDR-TB, DA prevalence varied widely and ranged from $25 \%$ to $47.1 \%$ of cases [17]. Currently, there are no completely reliable methods for the DA diagnostics [18]. Thus, DA and the immune mechanisms of DA in TB are a crucial problem of modern science $[19,20]$.

\section{Objective}

Detection of an AR to ATD in patients with MDR-TB.

\section{METHODS}

Study design: a retrospective study. To achieve the goal, the LLST was used to test the peripheral blood samples of MDR-TB patients. Clinical examination of 50 MDR-TB patients with suspected DA was carried out. The group of MDR-TB patients with signs of DA included $23 / 46.0 \%$ women and $27 / 54.0 \%$ men. The median age of the examined patients was 34.9 (with a range of 19-66 years). The patients were treated at the National Center for Phthisiology in 2016-2018. These patients received ATD according to standard treatment regimen. The laboratory method was used to determine type IV hypersensitivity or cell-mediated DA. This test is an in vitro provocation test. The test is safe for the patient, since application of the suspected allergen and the destruction of the patient's blood cells take place in vitro. Test was conducted for 2 hours of at $+37^{\circ} \mathrm{C}$. The result was assessed using light microscopy according to the degree of leukocyte lysis. Thus, the possibility of sensitizing the patient with particular drugs was assessed.

The results of the study by the LLST method were divided into three groups:

- Negative LLST (LLST-) - destruction of 0 to $10 \%$ of leukocytes during incubation of blood with an ATD.

- Doubtful (weakly positive) LLST (LLST \pm ) - destruction of more than $10 \%$, but less than $20 \%$ of leukocytes during incubation of blood with an ATD. 
Результаты исследования методом РСЛЛ разделены на три группы:

- Отрицательные РСЛЛ (РСЛЛ-) - разрушение от 0 до $10 \%$ лейкоцитов в процессе инкубации крови с противотуберкулёзным препаратом.

- Сомнительные (слабоположительные) РСЛЛ (РСЛЛ \pm ) - разрушение более $10 \%$, но менее $20 \%$ лейкоцитов в процессе инкубации крови с противотуберкулёзным препаратом.

- Положительные РСЛЛ (РСЛЛ+) - разрушение от $20 \%$ и более лейкоцитов в процессе инкубации крови с противотуберкулёзным препаратом.

Выполнена адаптация метода для выявления ЛА к ПТП. Для выявления IV типа ЛА использовался метод РСЛЛ к 9 препаратам: изониазиду (Н), пиразинамиду (Z), этамбутолу (Е), левофлоксацину (Lfx), циклосерину (Cs), протионамиду (Pt), каприомицину (Cm), ПАСК (PAS) и пиридоксину (В 6 ). Положительным считался результат РСЛЛ, когда у пациента была выявлена ЛА к одному препарату или более (до 9 ПтП).

Статистический анализ проводился методами вариационной статистики на ПК с использованием прикладного пакета SPSS 16.0. Для относительных величин определены частоты, вычислялись доли (\%). Для проверки значимости различий двух и более воздействий на группы, при наличии переменных с дихотомными вариантами, применялся Q-критерий Кохрена.

\section{РЕЗУЛЬТАТЫ И ИХ ОБСУЖДЕНИЕ}

По данным микроскопического исследования мазка мокроты при поступлении в стационар бактериовыделение выявлено у 40/80,0\% больных, и МБТ в мазке не обнаружены у 10/20,0\%.

В результате исследования реакции лейкоцитов на инкубацию с ПТП по замедленному типу гиперсенсибилизации положительный результат РСЛЛ был отмечен у 39/78,0\% больных МЛУ ТБ на один или более ПтП. Слабоположительные результаты на один или более ПТП по данному тесту выявлены у 10/20,0\% пациентов и отрицательный РСЛЛ ко всем препаратам - у 1/2,0\%.

Каждому больному МЛУ ТБ выполнялись анализы РСЛЛ на несколько из 9 ПтП. Всего по всем 9 препаратам для 50 обследованных больных МЛУ ТБ выполнено 331 исследование. Среди них положительных результатов было 88/26,6\%, слабоположительных -69/20,8\% и отрицательных - 174/52,6\%.

Таким образом, среди проведённых исследований одинаково часто встречались как положительные, так и отрицательные ре-

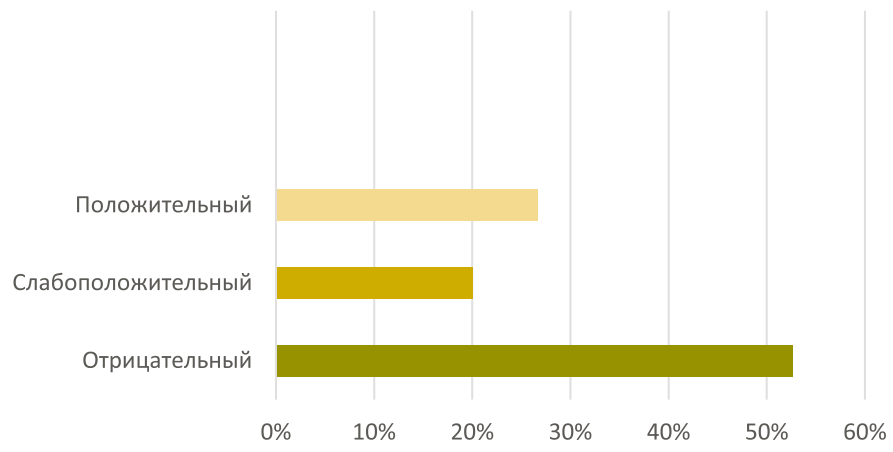

п 0\%-10\% РСлл- — 10\%-20\% РСлЛ $\pm 20 \%$ и более РСлЛ+

Рис. Результаты выявления аллергической реакции замедленного muna у больных МЛУ ТБ с использованием метода РСЛЛ
- $\quad$ Positive LLST (LLST+) - destruction of $20 \%$ or more leukocytes during incubation of blood with an ATD.

The applied method was adjusted for detecting of DA to ATD. To identify type IV hypersensitivity, we used the LLST method for 9 drugs: isoniazid $(H)$, pyrazinamide $(Z)$, ethambutol $(E)$, levofloxacin (Lfx), cycloserine (Cs), prothionamide (Pt), capreomycin $(\mathrm{Cm}), \mathrm{p}$-aminosalicylic acid (PAS) and pyridoxine $\left(\mathrm{B}_{6}\right)$. The LLST results were considered positive when the patient developed DA to one or more drugs (up to 9 ATD).

Statistical analysis was carried out by the methods of variation statistics using the SPSS 16.0 application package. For relative values, the frequencies were determined, and the proportions (\%) were calculated. To test the significance of differences between two or more treatments in the groups, in the presence of variables with dichotomous variants, the Cochran Q-test was used.

\section{RESULTS AND DISCUSSION}

According to microscopic examination of a sputum smear upon admission to the hospital, mycobacterium tuberculosis was detected in $40 / 80.0 \%$ of patients, while in the smears of another $10 / 20.0 \%$ of patients it was absent.

As a result of a study of the reaction of leukocytes to incubation with ATD on a delayed type of hypersensitization, a positive LLST was observed in 39/78.0\% of MDR-TB patients to one or more ATD. Weakly positive results for one or more ATD tested were found in $10 / 20.0 \%$ of patients, while negative LLST to all drugs - in $1 / 2.0 \%$.

Each MDR-TB patient underwent LLST for several out of the 9 anti-TB drugs. A total of 331 tests were performed on all 9 drugs for 50 examined MDR-TB patients. Among them, there were $88 / 26.6 \%$ with positive results, $69 / 20.8 \%$ - with weakly positive, and $174 / 52.6 \%$ with negative tests.

Thus, among the studies conducted, both positive and negative results were equally common $(47.4 \%$ and $52.6 \%$, respectively). The total number of tests with delayed-type AR (sum of RSLL+ and RSLL \pm ) was $157 / 47.4 \%$ (Fig.).

The number of studies on ATD with delayed-type hypersensitivity in MDR-TB patients is shown in Table 1.

Table 2 shows the number of delayed-type AR identified by the results of LLST.

The highest amount of type IV hypersensitivity AR was shown by CS (RSLL+ 16/35.6\% + RSLL $11 / 24.4 \%=27 / 60.0 \%$ ).

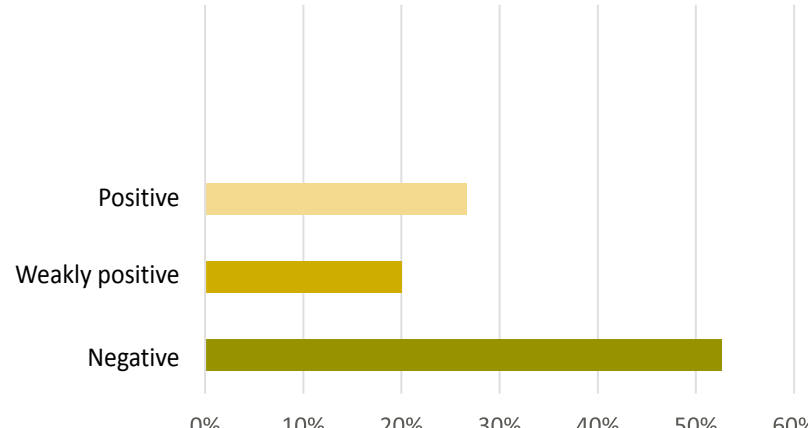

- 0\%-10\% LLST- $\quad 10 \%-20 \%$ LLST $\pm \quad 20 \%$ and more LLST+

Fig. Results of detecting of delayed-type AR in MDR-TB patients using the LLST method 
таблица 1 Количество РСЛЛ исследований (n) у больных МЛУ ТБ (распределение по препаратам)

\begin{tabular}{|c|c|c|c|c|c|c|}
\hline \multirow[t]{2}{*}{ птா } & \multicolumn{2}{|c|}{ Выявлено } & \multicolumn{2}{|c|}{ Не выявлено } & \multicolumn{2}{|c|}{ Всего } \\
\hline & n & $\%$ & n & $\%$ & $\mathbf{n}$ & $\%$ \\
\hline $\mathrm{H}$ & 11 & 22,0 & 39 & 78,0 & 50 & 100,0 \\
\hline Z & 45 & 90,0 & 5 & 10,0 & 50 & 100,0 \\
\hline $\mathrm{E}$ & 22 & 44,0 & 28 & 56,0 & 50 & 100,0 \\
\hline $\mathrm{B}_{6}$ & 31 & 62,0 & 19 & 38,0 & 50 & 100,0 \\
\hline Lfx & 46 & 92,0 & 4 & 8,0 & 50 & 100,0 \\
\hline Cs & 45 & 90,0 & 5 & 10,0 & 50 & 100,0 \\
\hline $\mathrm{Pt}$ & 48 & 96,0 & 2 & 4,0 & 50 & 100,0 \\
\hline $\mathrm{Cm}$ & 47 & 94,0 & 3 & 6,0 & 50 & 100,0 \\
\hline PAS & 36 & 72,0 & 14 & 28,0 & 50 & 100,0 \\
\hline
\end{tabular}

Table 1 Number of LLST tests ( $n$ ) in MDR-TB patients (distribution by drugs)

\begin{tabular}{|c|c|c|c|c|c|c|}
\hline \multirow[t]{2}{*}{ ATD } & \multicolumn{2}{|c|}{ Valid } & \multicolumn{2}{|c|}{ Missing } & \multicolumn{2}{|c|}{ Total } \\
\hline & $n$ & $\%$ & $n$ & $\%$ & $n$ & $\%$ \\
\hline H & 11 & 22.0 & 39 & 78.0 & 50 & 100.0 \\
\hline Z & 45 & 90.0 & 5 & 10.0 & 50 & 100.0 \\
\hline E & 22 & 44.0 & 28 & 56.0 & 50 & 100.0 \\
\hline $\mathrm{B}_{6}$ & 31 & 62.0 & 19 & 38.0 & 50 & 100.0 \\
\hline Lfx & 46 & 92.0 & 4 & 8.0 & 50 & 100.0 \\
\hline Cs & 45 & 90.0 & 5 & 10.0 & 50 & 100.0 \\
\hline Pt & 48 & 96.0 & 2 & 4.0 & 50 & 100.0 \\
\hline $\mathrm{Cm}$ & 47 & 94.0 & 3 & 6.0 & 50 & 100.0 \\
\hline PAS & 36 & 72.0 & 14 & 28.0 & 50 & 100.0 \\
\hline
\end{tabular}

Таблица 2 Аллергическая реакция на противотуберкулёзные препараты (метод реакции сенсибилизации лизиса лейкоцитов РСлЛ)

\begin{tabular}{|c|c|c|c|c|c|c|c|c|}
\hline \multirow{2}{*}{ птп } & \multicolumn{2}{|c|}{ РСЛЛ+ } & \multicolumn{2}{|c|}{ РСлЛ \pm} & \multicolumn{2}{|c|}{ РСлЛ+ РСлЛ \pm} & \multicolumn{2}{|c|}{ РСлл- } \\
\hline & $\mathbf{n}$ & $\%$ & $\mathbf{n}$ & $\%$ & $\mathrm{n}$ & $\%$ & $\mathbf{n}$ & $\%$ \\
\hline Cs & 16 & 35,6 & 11 & 24,4 & 27 & 60,0 & 18 & 40,0 \\
\hline H & 4 & 36,4 & 2 & 18,2 & 6 & 54,6 & 5 & 45,4 \\
\hline PAS & 11 & 30,6 & 8 & 22,2 & 19 & 52,8 & 17 & 47,2 \\
\hline Pt & 13 & 27,1 & 12 & 25,0 & 25 & 52,1 & 23 & 47,9 \\
\hline Lfx & 14 & 30,4 & 10 & 21,7 & 24 & 52,1 & 22 & 47,9 \\
\hline E & 4 & 18,2 & 5 & 22,7 & 9 & 40,9 & 13 & 59,1 \\
\hline $\mathrm{B}_{6}$ & 6 & 19,4 & 6 & 19,4 & 12 & 38,8 & 19 & 61,2 \\
\hline $\mathrm{Cm}$ & 11 & 23,4 & 7 & 14,9 & 18 & 38,3 & 29 & 61,7 \\
\hline Z & 9 & 20,0 & 8 & 17,8 & 17 & 37,8 & 28 & 62,2 \\
\hline
\end{tabular}

Table 2 AR to ATD (LLST)

\begin{tabular}{|c|c|c|c|c|c|c|c|c|}
\hline \multirow{2}{*}{ ATD } & \multicolumn{2}{|c|}{ LLST+ } & \multicolumn{2}{|c|}{ LLST \pm} & \multicolumn{2}{|c|}{ LLST+ LLST \pm} & \multicolumn{2}{|c|}{ LLST- } \\
\hline & $\mathbf{n}$ & $\%$ & $\mathrm{n}$ & $\%$ & $\mathbf{n}$ & $\%$ & $n$ & $\%$ \\
\hline Cs & 16 & 35.6 & 11 & 24.4 & 27 & 60.0 & 18 & 40.0 \\
\hline H & 4 & 36.4 & 2 & 18.2 & 6 & 54.6 & 5 & 45.4 \\
\hline PAS & 11 & 30.6 & 8 & 22.2 & 19 & 52.8 & 17 & 47.2 \\
\hline Pt & 13 & 27.1 & 12 & 25.0 & 25 & 52.1 & 23 & 47.9 \\
\hline Lfx & 14 & 30.4 & 10 & 21.7 & 24 & 52.1 & 22 & 47.9 \\
\hline E & 4 & 18.2 & 5 & 22.7 & 9 & 40.9 & 13 & 59.1 \\
\hline$B_{6}$ & 6 & 19.4 & 6 & 19.4 & 12 & 38.8 & 19 & 61.2 \\
\hline $\mathrm{Cm}$ & 11 & 23.4 & 7 & 14.9 & 18 & 38.3 & 29 & 61.7 \\
\hline Z & 9 & 20.0 & 8 & 17.8 & 17 & 37.8 & 28 & 62.2 \\
\hline
\end{tabular}


Таблица 3 Аллергическая реакция на противотуберкулёзные препараты ПТП (метод сенсибилизации лизиса лейкоцитов РСлЛ)

\begin{tabular}{|c|c|c|c|c|c|c|c|c|c|}
\hline \multirow{2}{*}{ птп } & \multicolumn{9}{|c|}{ Значение р } \\
\hline & Cs & H & PAS & Pt & Lfx & $E$ & $B_{6}$ & $\mathrm{Cm}$ & Z \\
\hline Cs & - & $<0,001$ & 0,202 & 0,762 & 0,538 & $<0,05$ & $<0,05$ & 0,147 & 0,104 \\
\hline H & $<0,001$ & - & $<0,05$ & $<0,001$ & $<0,001$ & 0,597 & 0,225 & $<0,05$ & $<0,05$ \\
\hline PAS & 0,202 & $<0,05$ & - & 0,330 & 0,508 & 0,075 & 0,186 & 0,861 & 0,724 \\
\hline Pt & 0,762 & $<0,001$ & 0,330 & - & 0,754 & $<0,05$ & $<0,05$ & 0,251 & 0,185 \\
\hline Lfx & 0,538 & $<0,001$ & 0,508 & 0,754 & - & $<0,05$ & 0,049 & 0,403 & 0,310 \\
\hline$E$ & $<0,05$ & 0,597 & 0,075 & $<0,05$ & $<0,05$ & - & 0,498 & 0,108 & 0,152 \\
\hline$B_{6}$ & $<0,05$ & 0,225 & 0,186 & $<0,05$ & 0,049 & 0,652 & - & 0,250 & 0,330 \\
\hline $\mathrm{Cm}$ & 0,147 & $<0,05$ & 0,861 & 0,251 & 0,403 & 0,108 & 0,250 & - & 0,858 \\
\hline Z & 0,104 & $<0,05$ & 0,724 & 0,185 & 0,310 & 0,152 & 0,330 & 0,858 & - \\
\hline
\end{tabular}

Table 3 AR to ATD (LLST)

\begin{tabular}{|c|c|c|c|c|c|c|c|c|c|}
\hline \multirow{2}{*}{ ATD } & \multicolumn{9}{|c|}{ p value } \\
\hline & Cs & $\mathbf{H}$ & PAS & Pt & Lfx & E & $B_{6}$ & $\mathrm{Cm}$ & Z \\
\hline Cs & - & $<0.001$ & 0.202 & 0.762 & 0.538 & $<0.05$ & $<0.05$ & 0.147 & 0.104 \\
\hline H & $<0.001$ & - & $<0.05$ & $<0.001$ & $<0.001$ & 0.597 & 0.225 & $<0.05$ & $<0.05$ \\
\hline PAS & 0.202 & $<0.05$ & - & 0.330 & 0.508 & 0.075 & 0.186 & 0.861 & 0.724 \\
\hline Pt & 0.762 & $<0.001$ & 0.330 & - & 0.754 & $<0.05$ & $<0.05$ & 0.251 & 0.185 \\
\hline Lfx & 0.538 & $<0.001$ & 0.508 & 0.754 & - & $<0.05$ & 0.049 & 0.403 & 0.310 \\
\hline$E$ & $<0.05$ & 0.597 & 0.075 & $<0.05$ & $<0.05$ & - & 0.498 & 0.108 & 0.152 \\
\hline $\mathrm{B}_{6}$ & $<0.05$ & 0.225 & 0.186 & $<0.05$ & 0.049 & 0.652 & - & 0.250 & 0.330 \\
\hline $\mathrm{Cm}$ & 0.147 & $<0.05$ & 0.861 & 0.251 & 0.403 & 0.108 & 0.250 & - & 0.858 \\
\hline Z & 0.104 & $<0.05$ & 0.724 & 0.185 & 0.310 & 0.152 & 0.330 & 0.858 & - \\
\hline
\end{tabular}

зультаты (47,4\% и 52,6\% соответственно). Общее число анализов с АР замедленного типа (сумма РСЛЛ+ и РСЛЛ士) оказалось равным $157 / 47,4 \%$ (рис.).

Количество исследований по ПТП на наличие гиперчувствительности замедленного типа у больных МЛУ ТБ представлено в табл. 1.

В табл. 2 представлено количество АР замедленного типа, выявленных по результатам РСЛЛ.

Самое высокое количество AP IV типа показал Cs (РСЛЛ+ $16 / 35,6 \%+$ РСЛЛ $\pm 11 / 24,4 \%=27 / 60,0 \%)$. Ha Pt $(25 / 52,1 \%)$, PAS (19/52,8\%), H (6/54,5\%) и Lfx (23/52,2\%) развитие AP было чаще, чем на другие ПтП, АР на Сm (18/38,3\%), Z (17/37,8\%), E (9/41,0\%) и $\mathrm{B}_{6}(12 / 38,7 \%)$ встречались примерно с одинаковой частотой.

Изучена частота развития АР (сумма РСЛЛ+ и РСЛЛ $)$ на отдельные препараты (табл. 3).

При анализе частоты АР у больных МЛУ ТБ на ПТП установлено, что с Н статистически значимое различие развития АР происходит чаще, чем с другими ПТП (Cm, Z, PAS p<0,05; Cs, Pt, Lfx p<0,001). Статистически значимые различия c Cs, Pt, Е встречались с одинаковой частотой $(p<0,05, p<0,001)$, но чаще чем c Lfx, $B_{6}(p<0,05$, $p<0,001)$ и с $C \mathrm{~m}, \mathrm{Z}$, PAS $(\mathrm{p}<0,05)$.

В ходе исследований выявлено, что у каждого обследованного больного может быть АР на один, два или большее число ПТП (табл. 4).
Development of AR was more common to such ATD as Pt (25/52.1\%), PAS (19/52.8\%), H (6/54.5\%) and Lfx (23/52.2\%). AR to $\mathrm{Cm}(18 / 38.3 \%), \mathrm{Z}(17 / 37.8 \%), \mathrm{E}(9 / 41.0 \%)$ and $\mathrm{B}_{6}(12 / 38.7 \%)$ occurred with approximately the same frequency.

The prevalence of AR (the sum of RSLL+ and RSLL \pm ) was studied for individual drugs (Table 3).

When analyzing the prevalence of AR in MDR-TB patients to ATD, it was found that a statistically significant difference in the development of AR was more common between $\mathrm{H}$ and such ATD as $\mathrm{Cm}, \mathrm{Z}$, PAS $(\mathrm{p}<0.05)$, and Cs, Pt, Lfx ( $p<0.001)$. Statistically significant differences with $\mathrm{Cs}, \mathrm{Pt}$, and $\mathrm{E}$ developed with the same frequency $(p<0.05, p<0.001)$, but more often than with $L f x, B_{6}$ $(p<0.05$ and $p<0.001$ respectively) and with $C m, Z$, PAS $(p<0.05)$.

It was discovered that each examined patient might have AR to one, two or more ATD (Table 4).

The most frequently observed were AR to the two ATD (12/24.0\% of patients); $9 / 18.0 \%$ of MDR-TB patients showed AR to the five and one ATD ( $p=0.624), 8 / 16.0 \%$ showed hypersensitivity to the three ATD $(p=0.454)$. In these cases, no statistically significant differences were found $(p>0.05)$. When comparing the prevalence of AR to the 2 ATD and to the 6 ATD (4/8.0\%), 4 ATD (3/6.0\%), 0 ATD, 7 ATD and 8 ATD (1/2.0\% each), statistically significant differences were obtained $(p<0.05)$. 
таблица 4 Наличие аллергической реакции, выявленной методом РСлЛ, к данному количеству ПтП

\begin{tabular}{|c|c|c|c|}
\hline \multirow{2}{*}{ Количество ПТП } & \multicolumn{2}{|c|}{ Аллергическая реакция } & \multirow{2}{*}{$\mathbf{p}$} \\
\hline & $\mathbf{n}$ & $\%$ & \\
\hline к 2 ПТП & 12 & 24,0 & \\
\hline к 1 ПТП & 9 & 18,0 & 0,624 \\
\hline к 5 ПТП & 9 & 18,0 & 0,624 \\
\hline к 3 ПтП & 8 & 16,0 & 0,454 \\
\hline к 6 ПтП & 4 & 8,0 & $<0,05$ \\
\hline к 4 ПТП & 3 & 6,0 & $<0,05$ \\
\hline к 0 ПтП & 1 & 2,0 & $<0,05$ \\
\hline к 7 ПТП & 1 & 2,0 & $<0,05$ \\
\hline к 8 ПТП & 1 & 2,0 & $<0,05$ \\
\hline
\end{tabular}

Table 4 Prevalence of AR detected by the LLST method to a given number of ATD

\begin{tabular}{|c|c|c|c|}
\hline Number of ATD & \multicolumn{2}{c|}{ AR } & \% \\
\hline 2 ATD & $\mathbf{n}$ & 24.0 \\
\hline ATD & 12 & 18.0 & 0.624 \\
\hline ATD & 9 & 18.0 & 0.624 \\
\hline ATD & 9 & 16.0 & 0.454 \\
\hline ATD & 8 & 8.0 & $<0.05$ \\
\hline ATD & 4 & 6.0 & $<0.05$ \\
\hline ATD & 3 & 2.0 & $<0.05$ \\
\hline ATD & 1 & 2.0 & $<0.05$ \\
\hline ATD & 1 & 2.0 & $<0.05$ \\
\hline
\end{tabular}

Наиболее часто наблюдались АР к 2 ПТП (12/24,0\% пациентов), по 9/18,0\% больных МЛУ ТБ показали АР к 5 ПТП и к 1 ПТП $(p=0,624)$, у 8/16,0\% отмечена гиперчувствительность к 3 ПТП $(p=0,454)$. В этих случаях статистически значимые различия не выявлены (p>0,05). При сравнении частоты АР к 2 ПТП и частот АР к 6 ПТП (4/8,0\%), 4 ПТП (3/6,0\%), 0 ПТП, 7 ПТП и 8 ПТП (1\2,0\%) выявлены статистически значимые различия $(p<0,05)$.

Проведено выявление ЛА (замедленной гиперчувствительности IV типа) с использованием метода РСЛЛ у 50 больных МЛУ ТБ на следующие препараты: H, Z, E, Lfx, Cs, Pt, Cm, PAS и пиридоксин $\left(\mathrm{B}_{6}\right)$. По одному или более препаратам РСЛЛ+ выявлен у 39/78,0\% обследованных на ЛА больных МЛУ ТБ с клиническими признаками ЛА, РСЛЛ - у 10/20,0\% и РСЛЛ- по всем исследованным препаратам - у 1/2,0\% больного МЛУ ТБ. Следовательно, из 50 больных, ЛА (реакция лизиса лейкоцитов при инкубации с ПТП) выявлена у 49/98,0\% из 50 обследованных больных МЛУ ТБ с подозрением на ЛА.

Наиболее аллергенным препаратом (по сумме РСЛЛ+ и РСлЛ士) является Cs (27/60,0\% лизиса лейкоцитов). Частота AP к 2 ПтП была наибольшей и составила 12/24,0\% пациентов.

Таким образом, среди больных МЛУ ТБ НЯ к ПТП наиболее чаще отмечаются к Cs $(27 / 60,0 \%)$, затем H $(6 / 54,5 \%)$ и, возможно, они носят токсико-аллергический характер. По литературным данным, АР встречались реже сравнительно с токсико-аллергическими реакциями. Так, частота развития токсических НЯ колеблется в пределах 24,5-62,8\% [21]. АР описаны в литературе преимущественно как кожные реакции (зуд, распространённая сыпь на коже) [22], что соответствует клиническим проявлениям обследованных пациентов МЛУ ТБ. По литературным данным, кожные реакции наблюдались у от 2,64\% до 37,5\% пациентов, пролеченных ПТП второго ряда [22, 23]. По другим данным, ЛА составляла от 25\% до 47,1\% случаев при терапии МлУ ТБ [17].
AR (type IV, delayed hypersensitivity) was identified using the LLST method in 50 MDR-TB patients to the following drugs: $H, Z, E, L f x, C s, P t, C m, P A S$ and pyridoxine $\left(B_{6}\right)$. For one or more drugs, RSLL+ was detected in 39/78.0\% of MDR-TB patients examined for AR with clinical signs of AR, RSLL \pm was found in $10 / 20.0 \%$ and RSLL-was observed for all studied drugs in $1 / 2.0 \%$ of MDRTB patients. Therefore, out of 50 patients, leukocyte lysis reaction during incubation with ATD was detected in 49/98.0\% of 50 examined MDR-TB patients with suspected AR.

The most allergenic drug (by the sum of RSLL+ and RSLL \pm ) is Cs (27/60.0\% of patients with leukocyte lysis). The prevalence of AR to the two ATD was the highest and amounted to $12 / 24.0 \%$ of patients.

Thus, among MDR-TB patients, AE to ATD, which might be of toxic allergic type, were most often reported to Cs (27/60.0\%), followed by $H(6 / 54.5 \%)$. According to the literature data, AR were less common in comparison with toxic allergic reactions. Thus, incidence of toxic AE ranges 24.5-62.8\% [21]. AR are described in the literature mainly as skin reactions (pruritus, disseminated skin rush) [22], which corresponds to the clinical manifestations seen in the examined MDR-TB patients. According to the literature, skin reactions were observed in $2.64 \%$ to $37.5 \%$ of patients treated with second-line ATD $[22,23]$. According to other data, DA accounted for $25 \%$ to $47.1 \%$ of MDR-TB cases [17].

\section{CONCLUSION}

Allergic reactions to ATD were detected in $49 / 98.0 \%$ of 50 examined MDR-TB patients with suspected AR based on the clinical data. The most allergenic drug was cycloserine (60\% patients with leukocyte lysis). The number of MDR-TB patients with an 


\section{ЗАКЛЮЧЕНИЕ}

Аллергические реакции на ПтП выявлены у 49/98,0\% из 50 обследованных больных МЛУ ТБ с подозрением на ЛА по клиническим данным. Наиболее аллергенным препаратом является циклосерин (60\% лизиса лейкоцитов). Количество больных МЛУ ТБ с аллергической реакцией к 2 ПтП было наибольшим и составило $12(24 \%)$ пациентов. allergic reaction to the two anti-TB drugs was the highest and amounted to $12(24 \%)$ patients.

\section{ЛИТЕРАТУРА}

1. Всемирная организация здравоохранения: Глобальный веб-сайт [Электронный ресурс]. 2020 [дата доступа: 2020 октябрь 4]; Режим доступа: http://who.int/ru/news-room/fact-sheets/detail/tuberculosis.

2. Aung KJM, Van Deun A, Declercq E, Sarker MR, Das PK, Hossain MA, et al. Successful "9-month Bangladesh regimen" for multidrug resistant tuberculosis among over 500 consecutive patients. Int J Tub Lung Dis. 2014;18:1188-94. Available from: https://doi.org/10.5588/ijtld.14.0100

3. Kuaban C, Noeske J, Rieder HL, Aït-Khaled N, Abena Foe JL, Trébucq A, et al. High effectiveness of a 12-month regimen for MDR-TB patients in Cameroon. Int J Tub Lung Dis. 2015;19(5):517-24. Available from: https://doi.org/10.5588/ ijtld.14.0535

4. Сводное руководство ВОЗ по лечению лекарственно-устойчивого туберкулёза. Копенгаген, Дания: Европейское региональное бюро ВОЗ; 2019. 120 c.

5. Марьяндышев АО, Лорсанов СМ, Хайдарханова ЗБ, Хункарсултанов СБ, Перхин ДВ, Свешникова ОМ, и др. Результаты применения деламанида в лечении туберкулёза с множественной и широкой лекарственной устойчивостью возбудителя в Российской Федерации. Туберкулёз и болезни лёгких. 2019;97(11):67-8. Available from: https://doi.org/10.21292/20751230-2019-97-11-67-68

6. Щегерцов ДЮ, Филинюк ОВ, Буйнова ЛН, Земляная НА, Кабанец НН, Аллилуев АС. Нежелательные побочные реакции при лечении больных туберкулёзом с множественной лекарственной устойчивостью возбудителя. Туберкулёз и болезни лёгких. 2018;96(3):35-43.

7. Gupta A, Kumar V, Natarajan S, Singla R. Adverse drug reactions and drug interactions in MDR-TB patients. Indian Journal of Tuberculosis. 2020;67(45):569-78. Available from: https://doi.org/10.1016/j. ijtb.2020.09.027

8. Павлова МВ, Старшинова АА, Сапожникова НВ, Чернохаева ИВ, Арчакова ли, Яблонский ПК. Эффективность комплексной терапии и возможные нежелательные реакции при лечении туберкулёза органов дыхания с множественной лекарственной устойчивостью возбудителя. Туберкулёз и болезни лёгких. 2015;12:61-7.

9. Dela Al, Tank ND, Singh AP, Piparva KG. Adverse drug reactions and treatment outcome analysis of DOTS-plus therapy of MDR-TB patients at district tuberculosis centre: A four year retrospective study. Lung India. 2017;34(6):522-6. Available from: https://doi.org/10.4103/0970.2113.217569

10. Mahata G, Rupam Kumar TA, Sen P, Mukhopadhyay S. A Study on adverse drug reaction profile of 2 nd line drugs in multi drug resistant (MDR) and extensively drug resistant (XDR) tuberculosis cases registered under DR-TB Centre in a Tertiary Care Hospital. J Evolution Med Dent Sci. 2020;9(5):280-3.

11. Вольф СБ. Нежелательные побочные реакции на химиотерапию туберкулёза. Журнал Гродненского государственного медицинского университета. 2016;3:141-5.

12. Шовкун ЛА, Кудлай ДЛ, Никитенко ИЮ, Кампос ЕД, Харсеева ГГ. Особенности формирования иммунного ответа при туберкулёзе с выделением лекарственно-чувствительных и лекарственно-устойчивых штаммов М. tuberculosis. Туберкулёз и болезни лёгких. 2019;97(6):44-9.

13. Лазаренко лЛ. Особенности диагностики лекарственной аллергии. Лабораторные методы исследования. Выпуск № 1. Санкт-Петербург, РФ: PRO Аллергодиагностика; 2015. 4 с.

\section{REFERENCES}

1. Vsemirnaya organizatsiya zdravookhraneniya: globalnyiy vebsayt [The World Health Organization: global web site] [Elektronnyiy resurs]. 2020 [data dostupa: 2020 oktyabr 4]; Rezhim dostupa: http://who.int/ru/news-room/factsheets/detail/tuberculosis.

2. Aung KJM, Van Deun A, Declercq E, Sarker MR, Das PK, Hossain MA, et al. Successful "9-month Bangladesh regimen" for multidrug resistant tuberculosis among over 500 consecutive patients. Int J Tub Lung Dis. 2014;18:1188-94. Available from: https://doi.org/10.5588/ijtld.14.0100

3. Kuaban C, Noeske J, Rieder HL, Aït-Khaled N, Abena Foe JL, Trébucq A, et al. High effectiveness of a 12-month regimen for MDR-TB patients in Cameroon. Int J Tub Lung Dis. 2015;19(5):517-24. Available from: https://doi.org/10.5588/ ijtld.14.0535

4. Svodnoe rukovodstvo VOZ po lecheniyu lekarstvenno-ustoychivogo tuberkulyoza [WHO consolidated guidelines on drug-resistant tuberculosis treatment]. Kopengagen: Evropeyskoe regional'noe byuro VOZ; 2019. 120 p.

5. Maryandyshev AO, Lorsanov SM, Khaydarkhanova ZB, Khunkarsultanov SB, Perkhin DV, Sveshnikova OM, i dr. Rezul'taty primeneniya delamanida v lechenii tuberkulyoza s mnozhestvennoy i shirokoy lekarstvennoy ustoychivost'yu vozbuditelya v Rossiyskoy Federatsii [Results of using delamanid in the treatment of multidrug-resistant and extensively drug-resistant tuberculosis in the Russian Federation]. Tuberkulyoz i bolezni lyogkikh. 2019;97(11):67-8. Available from: https://doi.org/10.21292/2075-1230-2019-97-11-67-68

6. Shchegertsov DYu, Filinyuk OV, Buynova LN, Zemlyanaya NA, Kabanets NN, Alliluev AS. Nezhelatelnye pobochnye reaktsii pri lechenii bolnykh tuberkulyozom s mnozhestvennoy lekarstvennoy ustoychivostyu vozbuditelya [Adverse events during treatment of patients suffering from multiple drug resistent tuberculosis]. Tuberkulyoz i bolezni lyogkikh. 2018;96(3):35-43.

7. Gupta A, Kumar V, Natarajan S, Singla R. Adverse drug reactions and drug interactions in MDR-TB patients. Indian Journal of Tuberculosis. 2020;67(45):56978. Available from: https://doi.org/10.1016/j.ijtb.2020.09.027

8. Pavlova MV, Starshinova AA, Sapozhnikova NV, Chernokhaeva IV, Archakova LI, Yablonskiy PK. Effektivnost' kompleksnoy terapii i vozmozhnye nezhelatelnye reaktsii pri lechenii tuberkulyoza organov dykhaniya $s$ mnozhestvennoy lekarstvennoy ustoychivosty'u vozbuditelya [Efficiency of integral therapy and potential side effects when treating respiratory tuberculosis with multiple drug resistance]. Tuberkulyoz i bolezni lyogkikh. 2015;12:61-7.

9. Dela Al, Tank ND, Singh AP, Piparva KG. Adverse drug reactions and treatment outcome analysis of DOTS-plus therapy of MDR-TB patients at district tuberculosis centre: A four year retrospective study. Lung India. 2017;34(6):522-6. Available from: https://doi.org/10.4103/0970.2113.217569

10. Mahata G, Rupam Kumar TA, Sen P, Mukhopadhyay S. A Study on adverse drug reaction profile of 2 nd line drugs in multi drug resistant (MDR) and extensively drug resistant (XDR) tuberculosis cases registered under DR-TB Centre in a Tertiary Care Hospital. J Evolution Med Dent Sci. 2020;9(5):280-3.

11. Volf SB. Nezhelatelnye pobochnye reaktsii na khimioterapiyu tuberkulyoza [Adverse drug reaction for chemotherapy tuberculosis]. Zhurnal Grodnenskogo gosudarstvennogo meditsinskogo universiteta. 2016;3:141-5.

12. Shovkun LA, Kudlay DL, Nikitenko IYu, Kampos ED, Kharseeva GG. Osobennosti formirovaniya immunnogo otveta pri tuberkulyoze s vydeleniem lekarstvenno-chuvstvitel'nykh i lekarstvenno-ustoychivykh shtammov $M$. tuberculosis [Specific features of the immune response to tuberculosis when drug susceptible and drug resistant strains of M. tuberculosis are detected]. Tuberkulyoz $i$ bolezni lyogkikh. 2019;97(6):44-9.

13. Lazarenko LL. Osobennosti diagnostiki lekarstvennoy allergii. Laboratornye metody issledovaniy. Vypusk № 1 [Features of the diagnosis of drug allergies. 
14. Новиков ПД, Новиков ДК, Титова НД. Диагностика аллергии и гиперчувствительности: ведущее значение клеточныхметодов. Иммунопатология, аллергология, инфектология. 2016;4:25-9.

15. Павлова МВ, Ершова ЕС, Чернохаева ИВ, Сапожникова НВ, Арчакова ЛИ. Нежелательные реакции при лечении туберкулёза органов дыхания препаратами нового поколения. Медицинский альянс. 2018;2:23-7.

16. Токтогонова АА. Частота и характер побочных реакций на противотуберкулёзные препараты второго ряда у больных туберкулёзом с множественной лекарственной устойчивостью возбудителя. Туберкулёз и болезни лёгких. 2017;95(10):63-7.

17. Rodina O, Borisov SE, Ivanova D. Adverse events in patients with MDR TB, treated by three types of the chemotherapy regimens. European Respiratory Journal. 2019;54(63):52-78. Available from: https://doi.org/10.1183/13993003. congress-2019.PA5278

18. Карпук ИЮ. Диагностика аллергии на местные анестетики в реакции антигениндуцированного повреждения лейкоцитов. Вестник Витебского государственного медицинского университета. 2010;9(1):1-8.

19. Cliff JM, Kaufmann SH, McShane H, van Helden P, O'Garra A. The human immune response to tuberculosis and its treatment: a view from the blood. Immunological Reviews. 2015;264(1):88-102. Available from: https://doi. org/10.1111/imr.12269

20. Thong BA, Chia F, Tan SC, Tan TC, Khai-Pang L, Wei-Lyn I, et al. Retrospective study on sequential desensitization-rechallenge for antituberculosis drug allergy. Asia Pacific Allergy. 2014;4(3):156-63. Available from: https://doi. org/10.5415/apallergy.2014.4.3.156

21. Buziashvili MG, Mirtskhulava V, Kipiani M, Blumberg HM, Baliashvili D, Magee $\mathrm{MJ}$, et al. Adverse reactions associated with injectable second-line antituberculosis drugs among patients with M/XDR-TB in Tbilisi. Int J Tuberc Lung Dis. 2019;23(9);1005-11. Available from: https://doi.org/10.5588/ijtld.18.0626

22. Rathod KB, Borkar MS, Lamb AR, Suryavanshi SL, Surwade GA, Pandey VR. Adverse events among patients of multi drug resistant tuberculosis receiving second line anti TB treatment. Journal International Scientific Report. 2015;1(6):253-7. Available from: https://doi.org/10.18203/issn.2454-2156. intjscirep20150955

23. Bezu JH, Seifu D, Yimer G, Mebrhatu T. Prevalence and risk factors of adverse drug reactions associated multidrug resistant tuberculosis treatments in selected treatment centers in Addis Ababa Ethiopia. Journal of Tuberculosis Research. 2014;(2):144-54. Available from: https://doi.org/10.4236/ jtr.2014.23018
Laboratory research methods. Issue 1]. Saint-Petersburg, RF: PRO Allergodiagnostika; 2015. 4 p.

14. Novikov PD, Novikov DK, Titova ND. Diagnostika allergii i giperchuvstvitel'nosti: vedushchee znachenie kletochnykh metodov [Diagnosis of allergies and hypersensitivity: the leading role of cell methods]. Immunopatologiya, allergologiya, infektologiya. 2016;4:25-9.

15. Pavlova MV, Ershova ES, Chernokhaeva IV, Sapozhnikova NV, Archakova LI. Nezhelatelnye reaktsii pri lechenii tuberkulyoza organov dykhaniya preparatami novogo pokoleniya [Adverse reactions during treatment of respiratory tuberculosis with drugs of a new generation]. Meditsinskiy al'yans. 2018;2:23-7.

16. Toktogonova AA. Chastota i kharakter pobochnykh reaktsiy na protivotuberkulyoznye preparaty vtorogo ryada u bol'nykh tuberkulyozom s mnozhestvennoy lekarstvennoy ustoychivost'yu vozbuditelya [Frequency and characteristics of adverse reactions to second line anti-tuberculosis drugs in patients with multiple drug resistant tuberculosis]. Tuberkulyoz i bolezni lyogkikh. 2017;95(10):63-7.

17. Rodina O, Borisov SE, Ivanova D. Adverse events in patients with MDR TB, treated by three types of the chemotherapy regimens. European Respiratory Journal. 2019;54(63):52-78. Available from: https://doi.org/10.1183/13993003. congress-2019.PA5278

18. Karpuk IYu. Diagnostika allergii na mestnye anestetiki v reaktsii antigenindutsirovannogo povrezhdeniya leykotsitov [Diagnosis of allergy to local anesthetics in the reaction of antigen-induced leukocyte damage]. Vestnik Vitebskogo gosudarstvennogo meditsinskogo universiteta. 2010;9(1):1-8.

19. Cliff JM, Kaufmann SH, McShane H, van Helden P, O'Garra A. The human immune response to tuberculosis and its treatment: a view from the blood Immunological Reviews. 2015;264(1):88-102. Available from: https://doi. org/10.1111/imr.12269

20. Thong BA, Chia F, Tan SC, Tan TC, Khai-Pang L, Wei-Lyn I, et al. Retrospective study on sequential desensitization-rechallenge for antituberculosis drug allergy. Asia Pacific Allergy. 2014;4(3):156-63. Available from: https://doi. org/10.5415/apallergy.2014.4.3.156

21. Buziashvili MG, Mirtskhulava V, Kipiani M, Blumberg HM, Baliashvili D, Magee $\mathrm{MJ}$, et al. Adverse reactions associated with injectable second-line anti-tuberculosis drugs among patients with M/XDR-TB in Tbilisi. Int J Tuberc Lung Dis. 2019;23(9);1005-11. Available from: https://doi.org/10.5588/ijtld.18.0626

22. Rathod KB, Borkar MS, Lamb AR, Suryavanshi SL, Surwade GA, Pandey VR. Adverse events among patients of multi drug resistant tuberculosis receiving second line anti TB treatment. Journal International Scientific Report. 2015;1(6):253-7. Available from: https://doi.org/10.18203/issn.2454-2156. intjscirep20150955

23. Bezu JH, Seifu D, Yimer G, Mebrhatu T. Prevalence and risk factors of adverse drug reactions associated multidrug resistant tuberculosis treatments in selected treatment centers in Addis Ababa Ethiopia. Journal of Tuberculosis Research. 2014;(2):144-54. Available from: https://doi.org/10.4236/ jtr.2014.23018

\section{(1) Сведения ОБ Авторах}

Майканаев Болот Бекташевич, доктор медицинских наук, директор, Национальный центр фтизиатрии

E-mail: maykanaev64@mail.ru

Токтогонова Атыркул Акматбековна, доктор медицинских наук, заместитель директора по науке, Национальный центр фтизиатрии

Scopus ID: 57193609315

ORCID ID: 0000-0002-6419-1032

SPIN-код: 2992-8520

E-mail: atyrkul7@gmail.com

Дуденко Елена Вячеславовна, старший научный сотрудник лаборатории иммунологии и молекулярной биологии, Национальный центр фтизиатрии

Researcher ID: AAD-5413-2021

Scopus ID: 55386919000

ORCID ID: 0000-0001-8948-3659

SPIN-код: 2716-2722

Author ID: 948002

E-mail: dudenko.e@list.ru

\section{(1) AUthor INFORMATION}

Maykanaev Bolot Bektashevich, Doctor of Medical Sciences, Director, National Center for Phthisiology

E-mail: maykanaev64@mail.ru

Toktogonova Atyrkul Akmatbekovna, Doctor of Medical Sciences, Deputy Director for Science, National Center for Phthisiology

Scopus ID: 57193609315

ORCID ID: 0000-0002-6419-1032

SPIN: $2992-8520$

Dudenko Elena Vyacheslavovna, Senior Researcher, Laboratory of Immunology and Molecular Biology, National Center for Phthisiology

Researcher ID: AAD-5413-2021

Scopus ID: 55386919000

ORCID ID: 0000-0001-8948-3659

SPIN: 2716-2722

Author ID: 948002

E-mail: dudenko.e@list.ru 
Сыдыкова Салтанат, научный сотрудник лаборатории иммунологии и молекулярной биологии, Национальный центр фтизиатрии

ORCID ID: 0000-0001-6479-2770

SPIN-код: 4398-8285

Author ID: 948012

E-mail: Saltanat.sydykova58@mail.ru

Информация об источнике поддержки в виде грантов, оборудования, лекарственных препаратов

Работа выполнялась в соответствии с планом НИР Национального центра фтизиатрии (№ государственной регистрации 0007719). Финансовой поддержки со стороны компаний-производителей лекарственных препаратов и медицинского оборудования авторы не получали

Конфликт интересов: отсутствует

\section{АДРЕС ДЛЯ КОРРЕСПОНДЕНЦИИ:}

Дуденко Елена Вячеславовна

старший научный сотрудник лаборатории иммунологии и молекулярной биологии, Национальный центр фтизиатрии

720020, Кыргызская Республика, г. Бишкек, ул. Ахунбаева, 90а Тел.: +996 (554) 044011

E-mail: dudenko.e@list.ru

\section{ВКЛАД АВТОРОВ}

Разработка концепции и дизайна исследования: МББ, ТАА

Сбор материала: ДЕВ, СС

Статистическая обработка данных: ТАA, ДЕВ, СС

Анализ полученных данных: ТАА, ДЕВ, СС

Подготовка текста: ДЕВ, СС

Редактирование: ТАА, ДЕВ

Общая ответственность: МББ
Sydykova Saltanat Sydykovna, Senior Researcher, Laboratory of Immunology and Molecular Biology, National Center for Phthisiology

ORCID ID: 0000-0001-6479-2770

SPIN: 4398-8285

Author ID: 948012

E-mail: Saltanat.sydykova58@mail.ru

\section{Information about support in the form of grants, equipment, medications}

The research was carried out in accordance with the research plan of the National Center for Phthisiology (state registration number - 0007719). The authors did not receive financial support from companies manufacturing medications and medical equipment

Conflicts of interest: The authors have no conflicts of interest

\section{ADDRESS FOR CORRESPONDENCE:}

Dudenko Elena Vyacheslavovna

Senior Researcher, Laboratory of Immunology and Molecular Biology, National Center for Phthisiology

720020, Kyrgyz Republic, Bishkek, Akhunbaeva str., 90a

Tel.: + 996 (554) 044011

E-mail: dudenko.e@list.ru

\section{AUTHOR CONTRIBUTIONS}

Conception and design: MBB, TAA

Data collection: DEV, SS

Statistical analysis: TAA, DEV, SS

Analysis and interpretation: TAA, DEV, SS

Writing the article: DEV, SS

Critical revision of the article: TAA, DEV

Overall responsibility: MBB

$\begin{array}{ll}\text { Submitted } & 25.02 .21 \\ \text { Accepted } & 25.06 .21\end{array}$

